\title{
AN IDENTITY FOR ELLIPTIC EQUATIONS WITH APPLICATIONS
}

\author{
BY \\ C. A. SWANSON( $\left.{ }^{1}\right)$
}

1. Introduction. An elementary identity involving a linear elliptic partial differential operator $L$ and its associated hermitian form will be used to obtain new comparison theorems, oscillation theorems, and lower bounds for eigenvalues. Comparison theorems will be obtained for both subsolutions and complex-valued solutions in unbounded domains of Euclidean space, generalizing earlier results of Hartman and Wintner [4], Protter [8], and the author [11], [12]. Oscillation theorems of Kreith's type [6] will be extended to (i) unbounded domains; (ii) nonself-adjoint operators; and (iii) subsolutions.

Lower bounds for the eigenvalues of $L$ arise naturally from the basic identity in the case of bounded domains, and are extended to unbounded domains when the coefficients of $L$ satisfy suitable conditions. The form of the lower bounds is the same as that obtained by Protter and Weinberger [9], [10] for bounded domains.

2. The main lemma. The linear elliptic differential operator $L$ defined by

$$
L v=\sum_{i, j=1}^{n} D_{i}\left(A_{i j} D_{j} v\right)+2 \sum_{i=1}^{n} B_{i} D_{i} v+C v
$$

will be considered on unbounded domains $R$ in $n$-dimensional Euclidean space $E^{n}$. The boundary $P$ of $R$ is supposed to have a piecewise continuous unit normal vector at each point. As usual, points in $E^{n}$ are denoted by $x=\left(x_{1}, x_{2}, \ldots, x_{n}\right)$ and differentiation with respect to $x_{i}$ is denoted by $D_{i}, i=1,2, \ldots, n$. The coefficients $A_{i j}, B_{i}$, and $C$ are assumed to be real and continuous in $R \cup P$ and the matrix $\left(A_{i j}\right)$ positive definite in $R$ (ellipticity condition). The domain $\mathfrak{D}_{L}=\mathfrak{D}_{L}(R)$ of $L$ is defined to be the set of all complex-valued functions $v \in C^{1}(R \cup P)$ such that all derivatives of $v$ involved in $L v$ exist and are continuous at every point in $R$.

Let $T_{a}$ denote the $n$-disk $\left\{x \in E^{n}:\left|x-x_{0}\right|<a\right\}$ and let $S_{a}$ denote the bounding $(n-1)$-sphere, where $x_{0}$ is a fixed point in $E^{n}$. Define

$$
R_{a}=R \cap T_{a}, \quad P_{a}=P \cap T_{a}, \quad C_{a}=R \cap S_{a} .
$$

Clearly there exists a positive number $a_{0}$ such that $R_{a}$ is a bounded domain with boundary $P_{a} \cup C_{a}$ for all $a \geqq a_{0}$.

Received by the editors July 3, 1967.

(1) Research sponsored by the Air Force Office of Scientific Research, Office of Aerospace Research, United States Air Force, under grant AF-AFOSR-379-67. 
Let $Q[z]$ be the hermitian form in $n+1$ variables $z_{1}, z_{2}, \ldots, z_{n+1}$ defined by

$$
Q[z]=\sum_{i, j=1}^{n} A_{i j} z_{i} \bar{z}_{j}-\sum_{i=1}^{n} B_{i}\left(z_{i} \bar{z}_{n+1}+z_{n+1} \bar{z}_{i}\right)+G\left|z_{n+1}\right|^{2}
$$

where $G$ is any continuous function in $R$ satisfying the inequality

$$
G \operatorname{det}\left(A_{i j}\right) \geqq \sum_{i=1}^{n} B_{i} B_{i}^{*},
$$

$B_{i}^{*}$ denoting the cofactor of $-B_{i}$ in the matrix associated with $Q[z]$. Condition (4) is known to be necessary and sufficient for $Q[z]$ to be positive semidefinite [2], [12].

Let $M_{a}$ be the quadratic functional defined by

where

$$
M_{a}[u]=\int_{R_{a}} F[u] d x
$$

$$
F[u]=\sum_{i, j} A_{i j} D_{i} u D_{j} \bar{u}-2 \operatorname{Re}\left(u \sum_{i} B_{i} D_{i} \bar{u}\right)+(G-C)|u|^{2} .
$$

Define $M[u]=\lim _{a \rightarrow \infty} M_{a}[u]$ (whenever the limit exists). The domain $\mathfrak{D}_{M}=\mathfrak{D}_{M}(R)$ of $M$ is defined to be the set of all complex-valued functions $u \in C^{1}(R \cup P)$ such that $M[u]$ exists and $u$ vanishes on $P$.

Define

$$
[u, v]_{a}=\int_{C_{a}} u \sum_{i, j} A_{i j} n_{i} D_{j} v d s
$$

where $\left(n_{i}\right)$ denotes the unit normal to $C_{a}$, and define

$$
[u, v]=\lim _{a \rightarrow \infty}[u, v]_{a},
$$

whenever the limit on the right side exists. The notation $M[u ; R]$ will be used for $M[u]$ and $[u, v ; R]$ will be used for $[u, v]$ in $\$ 5$ when different domains are under consideration.

An $L$-subsolution (-supersolution) is a real-valued function $v \in \mathscr{D}_{L}(R)$ which satisfies $L v \leqq 0(L v \geqq 0)$ at every point in $R$.

The following are extensions of results in [12] to subsolutions and supersolutions, and to complex-valued functions $u \in \mathfrak{D}_{M}(R)$.

LEMMA 1. For every $u \in C^{1}(R)$ and every real $v \in \mathfrak{D}_{L}(R)$ which does not vanish in $R$, the following identity is valid at each point in $R$ :

(9) $\sum_{i, 1} A_{i j} X_{i} \bar{X}_{j}-2 \operatorname{Re}\left(u \sum_{i} B_{i} \bar{X}_{i}\right)+G|u|^{2}+\sum_{i} D_{i}\left(|u|^{2} Y_{i}\right)=F[u]+|u|^{2} v^{-1} L v$,

where

$$
X_{i}=v D_{i}(u / v), \quad Y_{i}=v^{-1} \sum_{j=1}^{n} A_{i j} D_{j} v, \quad i=1,2, \ldots, n .
$$

The proof is a direct calculation similar to that given in [12]. 
THEOREM 1. If there exists $u \in \mathfrak{D}_{M}(R)$ not identically zero such that $M[u]<0$, then there does not exist an L-subsolution (-supersolution) $v$ satisfying $\left[|u|^{2} / v, v\right] \geqq 0$ which is positive (negative) everywhere in $R \cup P$. In particular, every real solution of $L v=0$ satisfying $\left[|u|^{2} / v, v\right] \geqq 0$ must vanish at some point of $R \cup P$. In the selfadjoint case $B_{i}=0, i=1,2, \ldots, n$, and $G=0$, the same conclusions are valid when the hypothesis $M[u]<0$ is weakened to $M[u] \leqq 0$.

Proof. Suppose to the contrary that there exists such a positive $L$-subsolution. Then integration of (9) over $R_{a}$ yields

$$
\int_{R_{a}} F[u] d x \geqq \int_{R_{a}} \sum_{i} D_{i}\left(|u|^{2} Y_{i}\right) d x
$$

since the first three terms on the left side of (9) constitute a positive semidefinite form by the hypothesis (4). Since $u=0$ on $P_{a}$, by the definition of $\mathscr{D}_{M}$, it follows from Green's formula that the right side of (10) is equal to

$$
\int_{P_{a} \cup C_{a}} \sum_{i}|u|^{2} n_{i} Y_{i} d s=\int_{C_{a}} \frac{|u|^{2}}{v} \sum_{i, j} A_{i j} n_{i} D_{j} v d s=\left[|u|^{2} / v, v\right]_{a} .
$$

Thus (7), (10), and the hypothesis $\left[|u|^{2} / v, v\right] \geqq 0$ imply that

$$
M[u]=\lim _{a \rightarrow \infty} \int_{R_{a}} F[u] d x \geqq 0 .
$$

The contradiction proves that a positive $L$-subsolution satisfying $\left[|u|^{2} / v, v\right] \geqq 0$ cannot exist. The analogous statement for a negative $L$-supersolution $v$ follows from the fact that $-v$ would then be a positive $L$-subsolution.

To prove the second statement of Theorem 1, suppose to the contrary that there exists a real solution $v \neq 0$ in $R \cup P$. Then $v$ would be either a positive $L$-subsolution or a negative $L$-supersolution in $R \cup P$.

The proof in the self-adjoint case is similar to that given in [12, p. 281] and will be omitted.

We remark that the condition $\left[|u|^{2} / v, v\right] \geqq 0$ of Theorem 1 is a mild "boundary condition at $\infty$ " generalizing the usual condition $v \neq 0$ on the boundary of bounded domains.

3. Lower bounds for eigenvalues. Let $\mathfrak{S}$ be the Hilbert space $\mathscr{L}^{2}(R)$, with inner product $\langle u, v\rangle=\int_{R} u(x) \bar{v}(x) d x$ and norm $\|u\|=\langle u, u\rangle^{1 / 2}$. Let $\mathscr{D}$ be the set of all complex-valued functions $u \in \mathscr{D}_{L} \cap \mathfrak{S}$ such that $u$ vanishes on $P$. In this section the elliptic operator (1), with domain $\mathfrak{D}$, is assumed to have the self-adjoint form

$$
L v=\sum_{i, j} D_{i}\left(A_{i j} D_{j} v\right)-C v,
$$

under the conditions described below (1). In the case of the Schrödinger operator $-L=-\Delta+C(x)$, it is well-known [1], [3, p. 146] that the lower part of the spectrum contains only eigenvalues of finite multiplicity if $C(x)$ is bounded from below. 
In the self-adjoint elliptic case, an assumption on the coefficients $A_{i j}$ is needed as well.

Let $A^{+}(x)$ denote the largest eigenvalue of $\left(A_{i j}(x)\right)$ and define

$$
\begin{aligned}
\alpha(r) & =\max _{1 \leqq|x| \leqq r} A^{+}(x), \\
\alpha_{0}(r) & =\max \left[\alpha(1), \max _{1 \leqq|x| \leqq r}|x|^{-2} A^{+}(x)\right],
\end{aligned}
$$

which are nondecreasing functions of $r$. The following assumptions are special cases of those given by Ikebe and Kato [5].

Assumptions. (i) $C(x)$ is bounded from below;

(ii) $\int_{1}^{\infty}\left[\alpha(r) \alpha_{0}(r)\right]^{-1 / 2}=\infty$.

It follows in particular from (i) and (ii) that the conditions $u \in \mathfrak{S}, L u \in \mathfrak{E}$ imply that $[u, u]=0[5]$.

Our purpose is to obtain a useful lower bound for the eigenvalues (if any) of $-L$. In the case of bounded domains, Protter and Weinberger [10] recently obtained results of this type by using a general form of the maximum principle. It will be shown here in the case of unbounded domains that a lower bound is available as an easy consequence of Lemma 1.

THEOREM 2. Let $\lambda$ be the lowest eigenvalue and $u$ be an associated normalized eigenfunction of the problem $-L u=\lambda u, u \in \mathfrak{D}$. If $v$ is any function in $\mathfrak{D}_{L}$ such that $v(x)>0$ for $x \in R \cup P$ and $\left[|u|^{2} / v, v\right] \geqq 0$, then

$$
\lambda \geqq \inf _{x \in R}[-L v(x) / v(x)] \text {. }
$$

Proof. With $B_{i}=0, i=1,2, \ldots, n$ and $G=0$, integration of (9) over $R_{a}$ yields

$$
M_{a}[u]+\int_{R_{a}}|u|^{2} v^{-1} L v d x \geqq \int_{R_{a}} \sum_{i} D_{i}\left(|u|^{2} Y_{i}\right) d x
$$

where the positive-definiteness of $\left(A_{i j}\right)$ has been taken into account. Since $u=0$ on $P_{a}$, it follows from Green's formula that

$$
\begin{aligned}
M_{a}[u] & =-\int_{R_{a}} \bar{u} L u d x+[u, u]_{a} \\
& =\lambda \int_{R_{a}}|u|^{2} d x+[u, u]_{a} .
\end{aligned}
$$

However, $\lim [u, u]_{a}=0(a \rightarrow \infty)$ is a general consequence of $u \in \mathfrak{S}$ and $L u \in \mathfrak{S}$ under the above assumptions [5], and therefore

$$
M[u]=\lim _{a \rightarrow \infty} M_{a}[u]=\lambda\|u\|^{2}=\lambda .
$$

As in the proof of Theorem 1, the right member of (12) has the limit $\left[|u|^{2} / v, v\right]$ as $a \rightarrow \infty$, which is nonnegative by hypothesis. Thus

which implies (11).

$$
\lambda+\int_{R}|u|^{2} v^{-1} L v d x \geqq 0,
$$


In the bounded case, the condition $\left[|u|^{2} / v, v\right] \geqq 0$ is vacuous and Theorem 2 reduces to a well-known result [9]. However, the proof given here is especially easy. We remark that the extra condition $\left[|u|^{2} / v, v\right] \geqq 0$ in the unbounded case is a condition on the asymptotic behavior of $v$ as $|x| \rightarrow \infty$; it is roughly equivalent to the usual hypotheses for bounded domains that $u=0$ on the boundary, $v>0$ in $R \cup P$, and $v \in C^{1}(R \cup P)$. In the case of the Schrödinger operator $-\Delta+C(x)$, it is known $\left[3\right.$, p. 179] that $|u(x)|<K e^{-\mu|x|}$, where $K$ and $\mu$ are constants, for every eigenfunction $u$, and hence various exponential functions can serve as the test functions $v$. As an easy example, consider the one-dimensional harmonic oscillator problem

$$
\begin{aligned}
-\frac{d^{2} u}{d x^{2}}+x^{2} u & =\lambda u, \quad 0 \leqq x<\infty \\
u(0) & =0
\end{aligned}
$$

The test function $v=\exp \left(-x^{2} / 2\right)$ yields the lower bound 1 whereas the exact lowest eigenvalue is known to be 3 .

4. Comparison theorems. Consider, in addition to (1), a second elliptic operator $l$ defined by

$$
l u=\sum_{i, j=1}^{n} D_{i}\left(a_{i j} D_{j} u\right)+2 \sum_{i=1}^{n} b_{i} D_{i} u+c u
$$

in which the coefficients satisfy the same conditions as the coefficients in (1). In addition to (5) consider the quadratic functional defined by

$$
m_{a}[u ; Q]=\int_{Q \cap T_{a}}\left[\sum_{i, j} a_{i j} D_{i} u D_{j} \bar{u}-2 \operatorname{Re}\left(u \sum_{i} b_{i} D_{i} \bar{u}\right)-c|u|^{2}\right] d x
$$

for every subdomain $Q \subset R$, and let $m[u ; Q]=\lim m_{a}[u ; Q](a \rightarrow \infty)$. The domain $\mathfrak{D}_{m}(Q)$ of $m$ is the analogue of $\mathfrak{D}_{M}(Q)$ (defined in $\S 2$ ). The variation of $L$ relative to the domain $Q$ is defined as $V[u ; Q]=m[u ; Q]-M[u ; Q]$, that is

$$
V[u ; Q]=\int_{Q}\left[\sum_{i, j}\left(a_{i j}-A_{i j}\right) D_{i} u D_{j} \bar{u}-2 \operatorname{Re}\left\{u \sum_{i}\left(b_{i}-B_{i}\right) D_{i} \bar{u}\right\}\right.
$$

with domain $\mathfrak{D}_{V}(Q)=\mathfrak{D}_{m}(Q) \cap \mathfrak{D}_{M}(Q)$.

$$
\left.+(C-c-G)|u|^{2}\right] d x
$$

The analogues of (7), (8) for the operator $l$ relative to the domain $Q$ are

$$
\begin{aligned}
\{u, v ; Q\}_{a} & =\int_{Q \cap s_{a}} \sum_{i, j} a_{i j} n_{i} \operatorname{Re}\left(u D_{j} \bar{v}\right) d s ; \\
\{u, v ; Q\} & =\lim _{a \rightarrow \infty}\{u, v ; Q\}_{a} .
\end{aligned}
$$

When $Q=R$ is the only domain under consideration, the abbreviations $V[u]$, $\{u, v\}$ will be used for $V[u ; R],\{u, v ; R\}$, respectively.

The following comparison theorems of Sturm's type are easy extensions of those 
in [12] to $L$-subsolutions (-supersolutions) and to complex-valued solutions of $l u=0$.

THEOREM 3. Suppose $G$ is a continuous function in $R$ satisfying the inequality (4). If there exists a nontrivial solution $u \in \mathscr{D}_{v}(R)$ of lu=0 such that $\{u, u\} \leqq 0$ and $V[u]>0$ then there does not exist an $L$-subsolution (-supersolution) which is positive (negative) everywhere in $R \cup P$ and satisfies $\left[|u|^{2} / v, v\right] \geqq 0$. In particular, every real solution of $L v=0$ satisfying $\left[|u|^{2} / v, v\right] \geqq 0$ must vanish at some point of $R \cup P$. The same conclusions hold if the hypotheses $V[u]>0,\left[|u|^{2} / v, v\right] \geqq 0$ are replaced by $V[u] \geqq 0$, $\left[|u|^{2} / v, v\right]>0$, respectively.

THEOREM 4. With $G$ as in Theorem 3 , if there exists a positive l-supersolution $u \in \mathfrak{D}_{V}(R)$ such that $\{u, u\} \leqq 0$ and $V[u]>0$, then the conclusions of Theorem 3 are valid.

Theorem 5 (Self-AdjoInt CASE). Suppose $b_{i}=B_{i}=0, i=1,2, \ldots, n$ in (1) and (13) and $G=0$. If there exists either (i) a nontrivial complex-valued solution $u \in \mathscr{D}_{v}(R)$ of $l u=0$, or (ii) a positive l-supersolution $u \in \mathscr{D}_{V}(R)$, such that $\{u, u\} \leqq 0$ and $V[u] \geqq 0$, then an L-subsolution (-supersolution) $v$ satisfying $\left[|u|^{2} / v, v\right] \geqq 0$ cannot be everywhere positive (negative) in $R \cup P$. In particular, every real solution of $L v=0$ satisfying $\left[|u|^{2} / v, v\right] \geqq 0$ must vanish at some point of $R \cup P$.

Proof of Theorem 3. Since $u=0$ on $P_{a}$, it follows from Green's formula that

$$
m_{a}[u]=-\int_{R_{a}} \operatorname{Re}(u l \bar{u}) d x+\{u, u\}_{a} .
$$

Since $l u=0$ and $l$ has real-valued coefficients, also $l \bar{u}=0$. Since $\{u, u\} \leqq 0$, we obtain in the limit $a \rightarrow \infty$ that $m[u] \leqq 0$. The hypothesis $V[u]>0$ is equivalent to $M[u]$ $<m[u]$. Hence $M[u]<0$ and Theorem 1 shows an $L$-subsolution (-supersolution) cannot be everywhere positive (negative) in $R \cup P$ under the hypothesis $\left[|u|^{2} / v, v\right]$ $>0$. The second statement of Theorem 3 also follows from Theorem 1 . The last statement follows upon obvious modifications of the inequalities.

If $u$ is a positive $l$-supersolution in $R$ such that $\{u, u\} \leqq 0$, it follows again from [17] that $m[u] \leqq 0$. The proof of Theorem 4 is then completed in the same way as that of Theorem 3. The proof of Theorem 5 follows similarly from the statement in Theorem 1 relative to the self-adjoint case.

It follows from (14) by partial integration that

where

$$
V[u ; Q]=\int_{Q}\left[\sum_{i, j}\left(a_{i j}-A_{i j}\right) D_{i} u D_{j} \bar{u}+\delta|u|^{2}\right] d x+\Omega(Q)
$$

and

$$
\delta=\sum_{i=1}^{n} D_{i}\left(b_{i}-B_{i}\right)+C-c-G
$$

$$
\Omega(Q)=\lim _{a \rightarrow \infty} \int_{Q \cap S_{a}} \sum_{i}\left(B_{i}-b_{i}\right)|u|^{2} n_{i} d s,
$$

whenever the limit exists. 
$L$ is called a strict Sturmian majorant of $l$ in $Q$ when the following conditions are fulfilled: (i) $\left(a_{i j}-A_{i j}\right)$ is positive semidefinite and $\delta \geqq 0$ in $Q$; (ii) $\Omega(Q) \geqq 0$; and (iii) either $\delta>0$ at some point in $Q$ or $\left(a_{i j}-A_{i j}\right)$ is positive definite and $c \neq 0$ at some point. A function defined in $Q$ is said to be of class $C^{2,1}(Q)$ when all of its second partial derivatives exist and are Lipschitzian in $Q$.

THEOREM 6. Suppose that $L$ is a strict Sturmian majorant of $l$ and that all the coefficients $a_{i j}$ involved in l are of class $C^{2,1}(R)$. If there exists a nontrivial solution $u \in \mathscr{D}_{v}(R)$ of lu=0 such that $\{u, u\} \leqq 0$, then no L-subsolution (-supersolution) $v$ satisfying $\left[|u|^{2} / v, v\right] \geqq 0$ can be everywhere positive (negative) in $R \cup P$. In particular, every real solution of $L v=0$ satisfying $\left[|u|^{2} / v, v\right] \geqq 0$ must vanish at some point of $R \cup P$.

TheOREM 7 (Self-ADJoINT CASE). Suppose $b_{i}=B_{i}=0, i=1,2, \ldots, n$ in (1) and (13), $G=0, C \geqq c$, and $\left(a_{i j}-A_{i j}\right)$ is positive semidefinite in $R \cup P$. If there exists either (i) a nontrivial complex-valued solution $u \in \mathfrak{D}_{v}(R)$ of $l u=0$, or (ii) a positive l-supersolution $u \in \mathscr{D}_{v}(R)$, such that $\{u, u\} \leqq 0$, then the conclusion of Theorem 6 is valid.

Since the pointwise conditions $G=0, C \geqq c$, and $\left(a_{i j}-A_{i j}\right)$ positive semidefinite obviously imply that $V[u] \geqq 0$, Theorem 7 is an immediate consequence of Theorem 5. The fact that the hypotheses of Theorem 6 imply $V[u]>0$ was demonstrated in [12, p. 283], and consequently the conclusion of Theorem 6 follows from Theorems 3 and 4.

In the special case of the Schrödinger operator $-l=-\Delta+c(x)$ with $c(x)$ bounded from below in $R$, the hypothesis $\{u, u\} \leqq 0$ of Theorems 5 and 7 can be replaced by $u \in \mathfrak{S}$ and $l u \in \mathfrak{S}$ since these conditions imply that $\{u, u\}=0[3, \mathrm{p}$. 56]. In the selfadjoint elliptic case, the same statement holds under quite general conditions on the coefficients, e.g. those stated prior to Theorem 2, as shown by Ikebe and Kato [5]. Also, the conclusion of Theorem 7 is valid even if $\left(A_{i j}\right)$ is only positive semidefinite provided $L$ is a strict Sturmian majorant of $l$ and all the coefficients $a_{i j}$ are of class $C^{2,1}(R)[12$, p. 283].

5. Oscillation theorems. In [6] Kreith obtained oscillation theorems for selfadjoint elliptic equations of the form $L v=0$ in the case that one variable $x_{n}$ is separable. He considered the case of bounded domains for which part of the boundary is singular. Here we shall obtain oscillation theorems of a general nature on unbounded domains by appealing to the comparison Theorems 3-7.

Let $T_{a}^{\prime}$ denote the complement of $T_{a}$ in $E^{n}$. A function $u$ is said to be oscillatory in $R$ at $\infty$, or simply oscillatory in $R$, whenever $u$ has a zero in $R \cap T_{a}^{\prime}$ for all $a>0$.

A domain (not necessarily bounded) $Q \subset R$ is called a nodal domain of a function $u$ iff $u=0$ on $\partial Q$ and $\{u, u ; Q\} \leqq 0$. If $Q$ is bounded, the latter condition is understood to be void, and the definition reduces to the standard definition of a nodal domain. If $-l$ is the Schrödinger operator with potential $c(x)$ bounded from below, sufficient 
conditions for $Q$ to be a nodal domain of $u \in D_{l}(Q)$ are $u=0$ on $\partial Q, u \in \mathfrak{E}$, and $l u \in \mathfrak{S}[3, \mathrm{p}$. 56]. A function $u$ is said to have the nodal property in $R$ whenever $u$ has a nodal domain $Q \subset R \cap T_{a}^{\prime}$ for all $a>0$.

The following results are immediate consequences of Theorems 3-7.

THEOREM 8. Suppose $G$ is a continuous function in $R$ satisfying (4). Suppose there exists either (i) a nontrivial complex-valued solution $u$ of $l u=0$, or (ii) $a$ positive $l$-supersolution $u$, with the nodal property in $R$ such that $V[u ; Q]>0$ for every nodal domain $Q$. Then every real solution of $L v=0$ is oscillatory in $R$ provided $\left[|u|^{2} / v, v ; Q\right]$ $\geqq 0$ for every $Q$. If the nodal domains are all bounded, every solution of $L v=0$ is oscillatory in $R$. In the self-adjoint case $b_{i}=B_{i}=0, i=1,2, \ldots, n$, the same conclusions hold under the weaker condition $V[u ; Q] \geqq 0$ for every nodal domain $Q$.

THEOREM 9. Suppose that $L$ is a strict Sturmian majorant of $l$ and that all the coefficients involved in $l$ are of class $C^{2,1}(R)$. If there exists a nontrivial complexvalued solution of $l u=0$ with the nodal property in $R$, then every real solution of $L v=0$ is oscillatory in $R$ provided $\left[|u|^{2} / v, v ; Q\right] \geqq 0$ for every nodal domain $Q$. If the nodal domains are all bounded, every solution of $L v=0$ is oscillatory in $R$. In the self-adjoint case $b_{i}=B_{i}=0, i=1,2, \ldots, n$, the same conclusions hold under the weaker hypotheses $G=0, C \geqq c$, and $\left(a_{i j}-A_{i j}\right)$ positive semidefinite in $R \cup P$.

Kreith has shown [6] that equations of the form

$$
D_{n}\left[a\left(x_{n}\right) D_{n} u\right]+\sum_{i, j=1}^{n-1} D_{i}\left[a_{i j}(\bar{x}) D_{j} u\right]+c\left(x_{n}\right) u=0, \quad \bar{x}=\left(x_{1}, x_{2}, \ldots, x_{n-1}\right),
$$

have bounded nodal domains in the form of cylinders, under suitable hypotheses, when $R$ is a bounded domain with an $(n-1)$-dimensional singular boundary. We shall show that the analogous construction for unbounded domains is valid provided $R$ is limit cylindrical, i.e. contains an infinitely long cylinder. Without loss of generality we can assume that $R$ contains a cylinder of the form

$$
G \times\left\{x_{n}: 0 \leqq x_{n}<\infty\right\},
$$

where $G$ is a bounded $(n-1)$-dimensional domain.

Let $\mu$ be the smallest eigenvalue of the boundary problem

$$
\begin{aligned}
-\sum_{i, j=1}^{n-1} D_{i}\left[a_{i j}(\bar{x}) D_{j} \phi\right] & =\mu \phi \quad \text { in } G, \\
\phi & =0 \text { on } \partial G .
\end{aligned}
$$

THEOREM 10. If there exists a positive number $b$ such that

$$
\int_{0}^{\infty} \frac{d t}{a(t)}=\infty \text { and } \int_{b}^{\infty}[c(t)-\mu] d t=\infty,
$$

then equation (18) has a solution $u$ with the nodal property in $R$. If $V[u ; Q] \geqq 0$ for every nodal domain $Q$, every solution of $L v=0$ is oscillatory in $R$. In particular, 
every solution of the self-adjoint equation $L v=0$ is oscillatory provided $C \geqq c$ and $\left(a_{i j}-A_{i j}\right)$ is positive semidefinite in $R \cup P$.

Proof. The hypotheses (20) imply that the ordinary differential equation

$$
D_{n}\left[a\left(x_{n}\right) D_{n} w\right]+\left[c\left(x_{n}\right)-\mu\right] w=0
$$

is oscillatory at $x_{n}=\infty$ on account of well-known theorems of Leighton [7] and Wintner [13]. Let $w$ be a solution with zeros at $x_{n}=\delta_{1}, \delta_{2}, \ldots, \delta_{m}, \ldots$, where $\delta_{m} \uparrow \infty$. If $\phi$ is an eigenfunction of (19) corresponding to the eigenvalue $\mu$, then the function $u$ defined by $u(x)=w\left(x_{n}\right) \phi(\bar{x})$ is a solution of (18) by direct calculation, with nodal domains in the form of cylinders

$$
G_{m}=G \times\left\{x_{n}: \delta_{m}<x_{n}<\delta_{m+1}\right\}, \quad m=1,2, \ldots
$$

Thus $u$ has a nodal domain $G_{m} \subset R \cap T_{a}^{\prime}$ for all $a>0$. In fact, given $a>0$, choose $m$ large enough so that $\delta_{m} \geqq a$. Then $x \in G_{m}$ implies $|x| \geqq\left|x_{n}\right|>a$ so $x \in T_{a}^{\prime}$. Hence (18) has a solution $u$ with the nodal property. The second statement of Theorem 10 follows from Theorem 8 and the last statement follows from Theorem 9.

\section{REFERENCES}

1. K. O. Friedrichs, Spektraltheorie halbbeschränkter Operatoren und Anwendung auf die Spektralzerlegung von Differentialoperatoren, Math. Ann. 109 (1934), 465-487.

2. F. R. Gantmacher, The theory of matrices, Vol. I, Chelsea, New York, 1959.

3. I. M. Glazman, Direct methods of qualitative spectral analysis of singular differential operators, Israel Program for Scientific Translations, Davey, New York, 1965.

4. Philip Hartman and Aurel Wintner, On a comparison theorem for self-adjoint partial differential equations of elliptic type, Proc. Amer. Math. Soc. 6 (1955), 862-865.

5. Teruo Ikebe and Tosio Kato, Uniqueness of the self-adjoint extension of singular elliptic differential operators, Arch. Rational Mech. Anal. 9 (1962), 77-92.

6. Kurt Kreith, Oscillation theorems for elliptic equations, Proc. Amer. Math. Soc. 15 (1964), 341-344.

7. Walter Leighton, The detection of the oscillation of solutions of a second order linear differential equation, Duke Math. J. 17 (1950), 57-62; On self-adjoint differential equations of second order, J. London Math. Soc. 27 (1952), 37-47.

8. M. H. Protter, A comparison theorem for elliptic equations, Proc. Amer. Math. Soc. 10 (1959), 296-299.

9. - Lower bounds for the first eigenvalue of elliptic equations, Ann. of Math. 71 (1960), 423-444.

10. M. H. Protter and H. F. Weinberger, On the spectrum of general second order operators, Bull. Amer. Math. Soc. 72 (1966), 251-255.

11. C. A. Swanson, A comparison theorem for elliptic differential equations, Proc. Amer. Math. Soc. 17 (1966), 611-616.

12. Comparison theorems for elliptic equations on unbounded domains, Trans. Amer. Math. Soc. 126 (1967), 278-285.

13. Aurel Wintner, A criterion of oscillatory stability, Quart. Appl. Math. 7 (1949), 115-117.

UNIVERSITY OF BRITISH COLUMBIA, VANCOUVER, B. C., CANADA 